\title{
Radical neck dissection: is it still indicated?
}

\author{
Marc Hamoir - Carl E. Silver - Sandra Schmitz - Robert P. Takes • \\ Alessandra Rinaldo $\cdot$ Juan P. Rodrigo $\cdot$ K. Thomas Robbins $\cdot$ Karen T. Pitman • \\ Jesus E. Medina $\cdot$ Alfio Ferlito
}

Received: 23 September 2012/ Accepted: 25 September 2012/Published online: 30 October 2012

(C) Springer-Verlag Berlin Heidelberg 2012

The presence of lymph node metastases in the neck remains a significant prognostic factor affecting patients with head and neck squamous cell carcinoma (HNSCC). Thus, treatment of regional disease remains a critical issue in the management of many patients.

The "classical" radical neck dissection (RND), consisting of removal of all the lymphatic and non-lymphatic structures from the mandible to the clavicle, with the exception of the carotid artery, hypoglossal, lingual, vagus and phrenic nerves, and brachial plexus, was developed in the late nineteenth and early twentieth century [1-4]. The procedure did not achieve widespread use in the medical community until the work of Martin et al. [5] when midcentury advances such as antibiotics, blood transfusion and modern anesthesia permitted safe and efficacious operation

This paper was written by members and invitees of the International Head and Neck Scientific Group (www.IHNSG.com).

M. Hamoir · S. Schmitz

Department of Head and Neck Surgery, St. Luc University

Hospital and Cancer Center, Université Catholique de Louvain,

Brussels, Belgium

C. E. Silver

Departments of Surgery and Otolaryngology-Head and Neck

Surgery, Albert Einstein College of Medicine,

Montefiore Medical Center, Bronx, NY, USA

R. P. Takes

Department of Otolaryngology-Head and Neck Surgery,

Radboud University Nijmegen Medical Center,

Nijmegen, The Netherlands

A. Rinaldo · A. Ferlito $(\bowtie)$

ENT Clinic, University of Udine,

Piazzale S. Maria della Misericordia, Udine 33100, Italy

e-mail: a.ferlito@uniud.it in a large series of patients. The classic RND has been and is still considered the "gold standard" for the surgical management of lymph node metastases of cancers of the head and neck.

There is little doubt that RND is oncologically a very effective surgical procedure, although beset with significant functional and cosmetic morbidity. During the second half of the twentieth century, a considerable evolution in the philosophy of surgical management of cervical lymph nodes in patients with head and neck cancer took place [6]. The wish to reduce the morbidity associated with RND, in particular regarding shoulder disability, gave impulse toward modifications of RND. The functional neck dissection of Suárez [7], which preserved the spinal accessory nerve (SAN), internal jugular vein (IJV), and sternocleidomastoid muscle (SCM), while removing the lymphatic structures in levels I or II through V, was developed and popularized [8] in the 1960s. Subsequently, Byers [9], at the MD Anderson Cancer Center, introduced the concept of

J. P. Rodrigo

Department of Otolaryngology,

Hospital Universitario Central de Asturias and Instituto

Universitario de Oncología del Principado de Asturias,

Oviedo, Spain

K. Thomas Robbins

Division of Otolaryngology-Head and Neck Surgery,

Southern Illinois University School of Medicine,

Springfield, IL, USA

K. T. Pitman

Department of Otolaryngology and Communicative Sciences,

University of Mississippi Medical Center, Jackson, MS, USA

\section{J. E. Medina}

Department of Otorhinolaryngology,

The University of Oklahoma Health Sciences Center,

Oklahoma City, OK, USA 
dissecting only the lymph nodes specifically at risk of metastases, according to the location of the primary tumor. Nevertheless, it was generally agreed, until recently, that RND was necessary for surgical management of advanced lymph node metastases of HNSCC. However, this concept is now challenged by the development of non-surgical treatment for advanced tumors and the emergence of less extended surgical procedures inducing less morbidity with equivalent oncologic outcome.

Over the past two decades, the concept of more tailored treatment, even for advanced disease in the neck, has gained increasing acceptance. The general application of this concept received a major impulse with the publications of the recommendations of the Committee for Head and Neck Surgery and Oncology of the American Academy of Otolaryngology-Head and Neck Surgery [10-12]. Wishing to respond to the need for uniformity, Robbins et al. [1012] proposed guidelines for the standardization of neck dissection with regard to terminology and extent of lymph node resection. In the proposed classification, RND was defined as the resection of lymph node levels $\mathrm{I}$ to $\mathrm{V}$, including removal of the SCM, IJV, and SAN. RND was considered the standard basic procedure for cervical lymphadenectomy while all other procedures represented one or more modifications of RND. Modified radical neck dissection (MRND) referred to the removal of all lymph nodes routinely resected by the RND but sparing one or more of the non-lymphatic structures (SAN, IJV, and SCM) usually removed during RND. Extended neck dissection was defined by the removal of one or more additional lymph node groups (e.g., parapharyngeal, paratracheal lymph nodes) or non-lymphatic structures (e.g., carotid artery paraspinal muscles, hypoglossal nerve, vagus nerve), not routinely resected by RND. Selective neck dissection (SND) refers to the preservation of one or more lymph node levels routinely resected during RND while sparing all non-lymphatic structures removed during RND. Recently a proposal for an even more rational and comprehensive classification has been proposed by Ferlito et al. [13] suggesting that the symbol ND for neck dissection be followed by the lymph node levels removed (I through VI) and the non-lymphatic structures resected (SCM, IJV, SAN, etc.).

Lymphatic metastases do not occur randomly, but in a predictable and well-documented pattern, in accordance with the site and subsite of the primary tumor, following the pre-existing lymphatic anatomy [14, 15]. Better knowledge of the anatomy of the lymphatics in the neck and improved understanding of lymphatic drainage patterns have led to surgical procedures which spare anatomical structures while removing the nodes at risk of containing metastasis. Application of this concept has significantly decreased the morbidity associated with the more radical procedures. Consequently, the trend toward increasing the frequency and indications for less radical procedures is justified.

At this point we may ask the following question: "If equivalent oncologic results can be accomplished with less morbidity by modified procedures, are there still indications for the use of RND?" Certainly RND is unacceptable for elective treatment of the clinically negative (N0) neck. Either SND or sentinel lymph node biopsy is the procedure of choice if elective treatment is chosen for the N0 neck. RND is also an overtreatment for most patients with clinically positive $(\mathrm{N}+)$ neck disease. There is increasing evidence suggesting that MRND [16] and even SND in patients with $\mathrm{N}+$ disease are equally efficacious as RND [17-20]. The SCM should be removed only when there is evidence of tumor extension or permeation of lymphatics. The SAN should be spared unless it is grossly infiltrated or encased by cancer. Even for patients with massive lymphadenopathy and significant extension to soft tissue, RND remains rarely indicated. Indeed, in this situation, when surgery is considered as primary treatment, it is rarely necessary to dissect all lymph node levels - although resection of one or more non-lymphatic structures or lymph node groups not usually included in RND may be necessary. In these circumstances the resultant disability could be very similar to that of classical RND or extended neck dissection, but resection of uninvolved tissue is avoided. For example, sublevel IA is usually not involved in patients with lymph node metastases from tumors of the larynx, hypopharynx, oropharynx, parotid gland, submandibular gland, thyroid gland, parathyroid gland, trachea, and cervical esophagus. Oral cavity SCCs do not usually metastasize to level $\mathrm{V}$. The apex of level $\mathrm{V}$ is never invaded in mucosal HNSCC and the dissection of this sublevel should only be considered in skin cancer of the posterior scalp and posterior neck [21]. Accordingly, the absence of metastases in sublevel IA and/or in sublevel VB does not justify a comprehensive dissection including levels I to $\mathrm{V}$ in patients with primary mucosal cancer of the head and neck [22, 23]. In 1992, Wei et al. [24] have reported that, in view of the usually extensive nature of neck lymph node metastasis of recurrent nasopharyngeal cancer, RND should be performed. However, in such a situation, neck dissection may require dissection of parapharyngeal lymph nodes not routinely resected in RND whereas the lymph nodes in sublevels IA and IB may be frequently spared, even in cases with advanced regional recurrence. Patients with persistent neck disease after concurrent chemoradiation for advanced N2 and N3 disease may benefit from SND in terms of regional disease control. Although nasopharyngeal carcinoma is a separate entity, it may be presumed that neck dissection should be tailored to the recurrence, following the same principles for salvage surgery of HNSCC as has been discussed above [25]. 
In patients with very advanced neck disease, more extended procedures than the classical RND are often required. These patients have a dismal prognosis due to the high-risk of distant metastasis as well as neck recurrence [26]. In current practice, there is a an emerging trend to treat those patients with chemoradiation, followed by neck dissection only in case of residual post-treatment disease in the neck [27]. When residual disease still persists in highrisk node levels following chemoradiotherapy, neck dissection is clearly required, but the likelihood of finding positive nodes in low-risk and initially uninvolved neck levels is still very low. Consequently, in HNSCC with advanced N2-N3 status treated by chemoradiation, SND and even super SND, limited to one or two levels, are suitable in most cases. Using this approach, complications are limited and oncologic outcomes are optimal [28-33].

The majority of patients who present with metastasis in the neck from an unknown primary tumor have advanced regional disease. Whereas the standard treatment for those patients has been RND followed by radiation therapy, there is now an increasing trend to treat such patients with a combination of chemotherapy and radiotherapy followed by post-treatment neck dissection in case of residual neck disease. Again, in this setting, RND is generally unnecessary and neck dissection should be limited to lymph node levels where residual disease is present [34].

In view of current concepts in head and neck oncology, we may conclude that the indications for RND are extremely limited [35, 36]. Accordingly, future updates to classification systems and publications on neck dissection should avoid consideration of RND as the "gold standard" for neck dissection, and should adopt a more rational approach.

\section{References}

1. Ferlito A, Johnson JT, Rinaldo A et al (2007) European surgeons were the first to perform neck dissection. Laryngoscope 117:797-802

2. Crile GW (1905) On the surgical treatment of cancer of the head and neck. With a summary of one hundred and twenty-one operations performed upon one hundred and five patients. Trans South Surg Gynecol Assoc 18:108-127

3. Ferlito A, Robbins KT, Silver CE (2010) Neck dissectionmanagement of regional disease in head and neck cancer. Plural Publishing, San Diego

4. Silver CE, Rinaldo A, Ferlito A (2007) Crile's neck dissection. Laryngoscope 117:1974-1977

5. Martin HE, Del Valle B, Ehrlich H, Cahan WG (1951) Neck dissection. Cancer 4:441-499

6. Ferlito A, Robbins KT, Shaha AR et al (2002) Current considerations in neck dissection. Acta Otolaryngol 122:323-329

7. Suárez O (1963) El problema de las metastasis linfáticas y alejadas del cáncer de laringe e hipofaringe. Rev Otorrinolaringol 23:83-99
8. Bocca E (1966) Supraglottic laryngectomy and functional neck dissection. J Laryngol Otol 80:831-838

9. Byers RM (1985) Modified neck dissection. A study of 967 cases from 1970 to 1980. Am J Surg 150:414-421

10. Robbins KT, Medina JE, Wolfe GT et al (1991) Standardizing neck dissection terminology. Official report of the Academy's Committee for Head and Neck Surgery and Oncology. Arch Otolaryngol Head Neck Surg 117:601-605

11. Robbins KT, Clayman G, Levine PA et al (2002) Neck dissection classification update: revisions proposed by the American Head and Neck Society and the American Academy of Otolaryngology-Head and Neck Surgery. Arch Otolaryngol Head Neck Surg 128:751-758

12. Robbins KT, Shaha AR, Medina JE et al (2008) Consensus statement on the classification and terminology of neck dissection. Arch Otolaryngol Head Neck Surg 134:536-538

13. Ferlito A, Robbins KT, Shah JP et al (2011) Proposal for a rational classification of neck dissections. Head Neck 33:445450

14. Lindberg R (1972) Distribution of cervical lymph node metastases from squamous cell carcinoma of the upper respiratory and digestive tracts. Cancer 29:1446-1449

15. Shah JP (1990) Patterns of cervical lymph node metastasis from squamous carcinomas of the upper aerodigestive tract. Am J Surg 160:405-409

16. Andersen PE, Shah JP, Cambronero E, Spiro RH (1994) The role of comprehensive neck dissection with preservation of the spinal accessory nerve in the clinically positive neck. Am J Surg 168:499-502

17. Pellitteri PK, Robbins KT, Neuman T (1997) Expanded application of selective neck dissection with regard to nodal status. Head Neck 19:260-265

18. Schmitz S, Machiels JP, Weynand B, Gregoire V, Hamoir M (2009) Results of selective neck dissection in the primary management of head and neck squamous cell carcinoma. Eur Arch Otorhinolaryngol 266:437-443

19. Patel RS, Clark JR, Gao K, O'Brien CJ (2008) Effectiveness of selective neck dissection in the treatment of the clinically positive neck. Head Neck 30:1231-1236

20. Robbins KT, Ferlito A, Shah JP et al (2012) The evolving role of selective neck dissection for head and neck squamous cell carcinoma. Eur Arch Otorhinolaryngol. (Epub ahead of print)

21. Hamoir M, Shah JP, Desuter G et al (2005) Prevalence of lymph nodes in the apex of level V: a plea against the necessity to dissect the apex of level $\mathrm{V}$ in mucosal head and neck cancer. Head Neck 27:963-969

22. Ferlito A, Kowalski LP, Silver CE, Shaha AR, Rinaldo A, Byers RM (2002) The use and misuse of level IA dissection for head and neck cancer. Acta Otolaryngol 122:553-555

23. Ferlito A, Rinaldo A (2008) Is radical neck dissection a current option for neck disease? Laryngoscope 118:1717-1718

24. Wei WI, Ho CM, Wong MP, Ng WF, Lau SK, Lam KH (1992) Pathological basis of surgery in the management of postradiotherapy cervical metastasis in nasopharyngeal carcinoma. Arch Otolaryngol Head Neck Surg 118:923-929

25. Khalif A, Ferlito A, Takes RP, Robbins KT (2010) Is it necessary to perform radical neck dissection as a salvage procedure for persistent or recurrent neck disease after chemoradiotherapy in patients with nasopharyngeal cancer? Eur Arch Otorhinolaryngol 267:997-999

26. Ferlito A, Silver CE, Shaha AR, Rinaldo A (2002) Management of N3 neck. Acta Otolaryngol 122:230-233

27. Hamoir M, Ferlito A, Schmitz S et al (2012) The role of neck dissection in the setting of chemoradiation therapy for head and neck squamous cell carcinoma with advanced neck disease. Oral Oncol 48:203-210 
28. Robbins KT, Wong FS, Kumar P et al (1999) Efficacy of targeted chemoradiation and planned selective neck dissection to control bulky nodal disease in advanced head and neck cancer. Arch Otolaryngol Head Neck Surg 125:670-675

29. Stenson KM, Haraf DJ, Pelzer H et al (2000) The role of cervical lymphadenectomy after aggressive concomitant chemoradiotherapy: the feasibility of selective neck dissection. Arch Otolaryngol Head Neck Surg 126:950-956

30. Robbins KT, Doweck I, Samant S, Vieira F (2005) Effectiveness of superselective and selective neck dissection for advanced nodal metastases after chemoradiation. Arch Otolaryngol Head Neck Surg 131:965-969

31. Stenson KM, Huo D, Blair E et al (2006) Planned post-chemoradiation neck dissection: significance of radiation dose. Laryngoscope 116:33-36

32. Robbins KT, Shannon K, Vieira F (2007) Superselective neck dissection after chemoradiation: feasibility based on clinical and pathologic comparisons. Arch Otolaryngol Head Neck Surg 133:486-489

33. Robbins KT, Dhiwakar M, Vieira F, Rao K, Malone J (2012) Efficacy of super-selective neck dissection following chemoradiation for advanced head and neck cancer. Oral Oncol. (Epub ahead of print)

34. Strojan P, Ferlito A, Langendijk JA et al (2011) Contemporary management of lymph node metastases from an unknown primary to the neck: II. A review of therapeutic options. Head Neck. (Epub ahead of print)

35. Ferlito A, Rinaldo A, Silver CE et al (2006) Elective and therapeutic selective neck dissection. Oral Oncol 42:14-25

36. Hamoir M, Leemans CR, Dolivet G, Schmitz S, Grégoire V, Andry G (2010) Selective neck dissection in the management of the neck after (chemo)radiotherapy for advanced head and neck cancer. Proposal for a classification update. Head Neck $32: 816-819$ 